\title{
Performance Evaluation of Video Streaming over Ad-Hoc Networks using Flat and Hierarchical Routing Protocols
}

\author{
Pau Arce \\ Juan Carlos Guerri \\ iTEAM (Institute of Telecommunications and Multimedia Applications) \\ University Polytechnic of Valencia \\ Ana Pajares \\ (a)
}

(Spain)

\author{
Óscar Lázaro \\ Innovalia Association
}

(Spain)

\begin{abstract}
A performance evaluation of real time services (such as video streaming) over mobile ad-hoc networks using both hierarchical and flat routing protocols is showed in this paper. A variety of workload and scenarios, as characterized by mobility, load and size of the ad hoc network have been simulated using NS-2. We use OLSR as the flat routing protocol and our own implementation of HOLSR (which uses HNA messages) as the hierarchical one. We make a complete comparison between these protocols focused on video evaluation. The simulations lets us compare the quality of service (QoS) of the video streaming paying attention to objective parameters (PSNR, packet delivery ratio, packet delay). Results indicate that traffic overload is reduced and video quality is improved using hierarchical protocols.
\end{abstract}

\section{Categories and Subject Descriptors}

C.2.1 [Network Architecture and Design]: Network communications, Wireless communication.

\section{General Terms}

Performance, Experimentation

\section{Keywords}

AdHoc networks, videostreaming, routing protocols, performance.

\section{INTRODUCTION}

Wireless technology has experienced a great growth in the past decade. The main advances can be found in network infrastructure, wireless applications development and wireless devices. We can now find a variety of these, such as mobile phones, PDAs and laptops, which are capable of sending and receiving real-time information like video.

Nowadays, a great interest is focused on mobile ad hoc networks (MANETs) (Figure 1). MANETs are formed by mobile nodes which are connected via wireless links without using an existing network infrastructure. Thus, MANETs don't require any fixed infrastructure such as a base station to operate. Moreover, routes between nodes may include multiple hops -that is why these networks are called multihop wireless ad hoc networks- because in order to communicate with nodes that are out of its transmission range, these nodes need to use intermediate nodes as routers [1], [2].

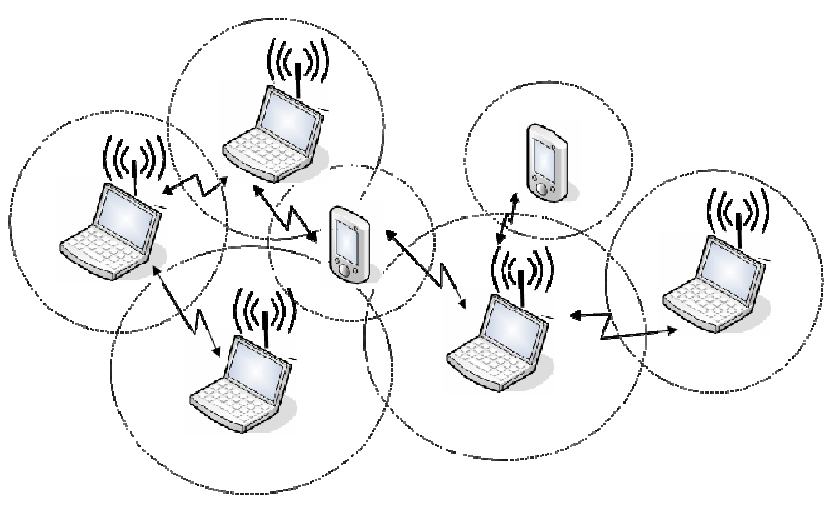

Figure 1. Example of a MANET.

Because of the dynamic topology of MANETs, routing protocols are more complex than traditional routing protocols used on the Internet. Anyway, the main objective of these routing protocols is achieving efficient routes between the nodes so that the information will be available in destination nodes reliably and within boundary time. A good performance of these protocols should have low overhead and bandwidth consumption, and a fast route convergence, even when there are changes in traffic load or the number of nodes (scalability).

A lot of works has been done on the routing protocols in ad hoc networks, taking into account different scenarios and traffic conditions [3], [4], [5]. Most of the routing protocols proposed consider ad hoc networks as an homogeneous one; that is that all nodes have the same capabilities. This kind of protocols is known as flat protocols [6], [7], [8], [9],[10] .

However, many ad hoc networks may be considered heterogeneous because there are mobile nodes with different capacities (bandwidth, transmission range, etc.). To maintain scalability in these heterogeneous ad hoc networks (capacity of a network to maintain its performance when the number of nodes increases) hierarchical routing protocols (Figure 2) should be considered as a good option [11], [12], [13]. 


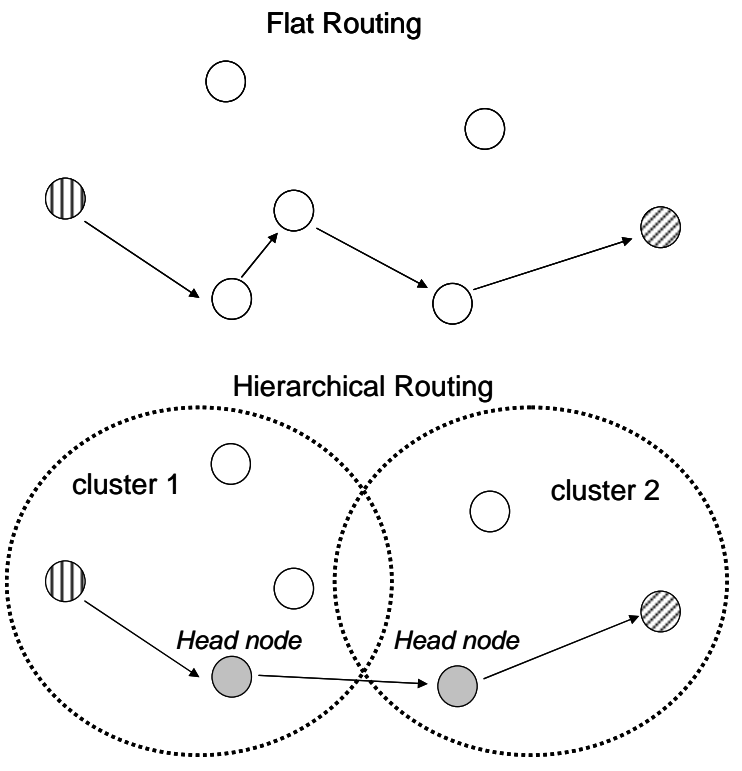

Figure 2. Flat and hierarchical routing protocols.

On the other hand, if the objective of the MANET is offering realtime services -such as video streaming- it will be interesting to evaluate the performance offered by the routing protocols. As performance metric it is important to pay attention to PSNR, packet delivery ratio and subjective quality of reconstructed videos [14].

This paper focuses on measuring and comparing video delivering quality over different ad hoc routing protocols (OLSR and HOLSR) using Evalvid tool integrated into NS-2.

The rest of this paper is organized as follows. Section 2 briefly compares the two routing protocols: OLSR and HOLSR. In Section 3 and Section 4 we show the simulation experiments and the simulation results of the routing and video performance evaluation respectively. Finally, we present the conclusions and our future work in Section 5.

\section{PROTOCOLS DESCRIPTION}

Both OLSR and HOLSR are routing proactive protocols. The main characteristic of proactive protocols is that each node in the network maintains a route to every other node in the network all the time.

In proactive approaches each node steadily keeps and updates a route to every node in the ad hoc network. So, routes are always available when they are needed. As a consequence there is a constant overhead due to routing traffic, but there is no initial delay in data communications. This constant overhead could become a disadvantage in large ad hoc networks or in ad hoc networks with a high mobility in the nodes.

\subsection{OLSR}

The Optimized Link State Protocol (OLSR) is described in RFC 3626 [6]. It is a variation of traditional link state routing, modified for improved operation in ad hoc networks. The optimization is based on a technique called MultiPoint Relaying (MPR).

OLSR defines two basic types on control messages:

- $\quad$ HELLO - They are transmitted to all neighbors.

- $\quad$ TC (Topology Control) - They diffuse topology information to their neighbors. This mechanism of diffusion is optimized using MPRs.

- $\quad$ HNA (Host and Network Association) - They are used by a host to announce itself as a gateway to specific networks.

A node sends a HELLO message to identify itself and it also contains a list of neighboring mobile nodes. From a HELLO message, the mobile node receives information about its immediate neighbors and two-hop neighbors. In these messages nodes also announce their own availability to act as MPR. There are 8 levels of willingness, from the lowest (indicates that this node must never be chosen as an MPR), to the highest (indicates that this node should always be chosen as an MPR). The willingness must be considered when calculating MPRs. RFC 3626 proposes a simple method for optimize MPR calculation.

A TC message is generated by the MPR nodes, announcing who has selected them as MPRs. Such messages are relayed by other MPRs throughout the whole network, enabling the remote nodes to discover the links between an MPRs and its selectors. Based on such information, the routing table is calculated using the shortest-path algorithm.

The OLSR protocol supports nodes having multiple interfaces. However, OLSR employs a "flat" mechanism, whereby a node sends HELLO and TC messages through all its interfaces without regard to the link capabilities of the other nodes. Thus, the flat OLSR mechanism does not scale well for large heterogeneous ad hoc networks.

\subsection{HOLSR}

Hierarchical OLSR is introduced to increase scalability of OLSR to large ad hoc networks. The basic principle in our hierarchical OLSR approach is similar to the CRC HOLSR described in [12] and [13] but there are several differences. In our proposal, we consider that there are only two levels in the hierarchy, where level-1 corresponds to connection among type- 1 nodes (core network) and level-2 corresponds to connection among type-2 nodes (access network) (Figure 3). An access subnetwork which is connected to other access subnetworks is hereafter referred to as a cluster. A type- 1 node serves as the cluster head and advertises its reachability to other clusters. The cluster heads are predefined, so there is no need to develop an algorithm for cluster head selection. In addition, the cluster heads are aware of each other, and are connected to each other, either directly or via multihop relays. Communications between cluster heads can also be conducted using unicast traffic, as an alternative to subnetdirected-broadcast. As in CRC HOLSR, the cluster nodes should have at least two wireless interfaces, for inter- and intra-cluster communications respectively. A directional antenna or more 
transmitting power is supposed to be used for inter-cluster connection. The data rate for inter-cluster connection should be higher than the ones for intra-cluster communication.

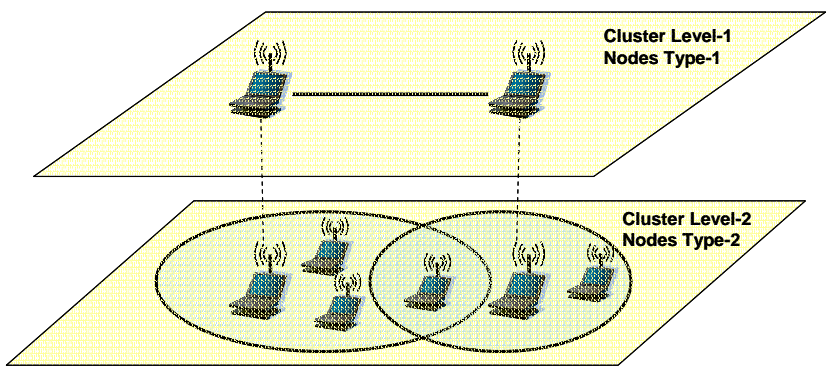

Figure 3. Hierarchical Ad Hoc Network

A major difference between our approach and CRC HOLSR is that we use only HNA messages for both inter-cluster and intracluster topology dissemination, while hierarchical TC messages are used for inter-cluster topology dissemination in CRC HOLSR. In other words, no modifications to HELLO and TC messages, or MPR selection, are foreseen with our approach.

According to the ideas described above, our HOLSR solution will be implemented based on only the modifications of HNA messages. Furthermore, it is the cluster head's responsibility to advertise its reachability to both internal nodes and other clusters.

To evaluate the performance of the proposed hierarchical OLSR solution, a flat OLSR network with the same number of nodes should also be established. The NS-2 implementation of our proposal should be able to demonstrate how the proposed solution works in terms of connectivity, scalability and quality of real-time services. Furthermore, performance evaluation will show that the protocol overhead for the hierarchical network is much less than the flat network of the same size.

\section{ROUTING PERFORMANCE EVALUATION}

\subsection{Performance metrics}

The performance of the OLSR and HOLSR protocols is evaluated in terms of the following parameters:

- Convergence Time: time spent by the routing protocol to stabilize the routing information in all wireless nodes (in seconds).

- Control Overhead: amount of control packets transmitted by the routing protocols (in packets/s).

Both parameters evaluate the efficiency of the routing protocols independently of the type of traffic.

The radio model used for simulation is based on the Two-Ray Ground Propagation Model and the standard 802.11b. The traffic model used to gather the numerical results consists of constant bit rate (CBR) sources defined over 20 communication links among nodes randomly selected. Each communication session consists of two UDP packets, 512 bytes long, being transmitted every second. The control overhead is measured in terms of the amount of control messages generated by the routing algorithm. In order to obtain these results, 20 simulations of each scenario have been done and mean results have been presented. Every simulation takes 30 seconds and nodes are statically distributed. Nodes in the HOLSR simulation are distributed within two clusters of size 400 $\mathrm{x} 400 \mathrm{~m}$. with the head nodes in the middle connected by a direct wireless link. For the OLSR simulation, the scenario size is $700 \mathrm{x}$ $400 \mathrm{~m}$.

\subsection{Simulation results}

In this section, we will report the results of the simulation experiments we have used to evaluate the performance of the ad hoc routing protocols.

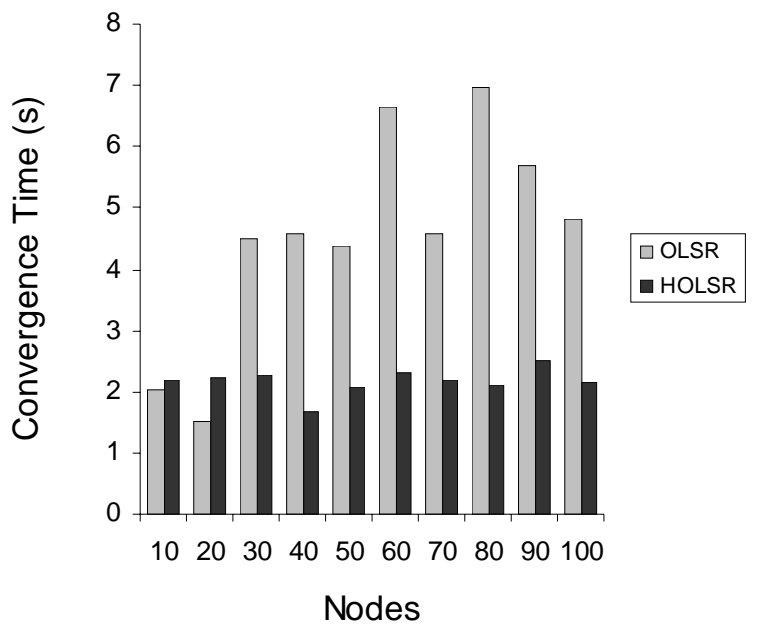

Figure 4. Convergence time for OLSR and HOLSR protocols.

Figure 4 shows that the time used by the OLSR protocol until it achieves stability (that is to say when every node from the network knows the route to arrive to every other node) varies deeply depending on the number of nodes which form that network. However, the HOLSR protocol keeps this time more or less constant until it reaches stabilization. That is due to the fact that the stability of a cluster is achieved quite soon and at the same time the cluster heads exchange information among them (through HNA messages) about nodes that belong to their cluster.

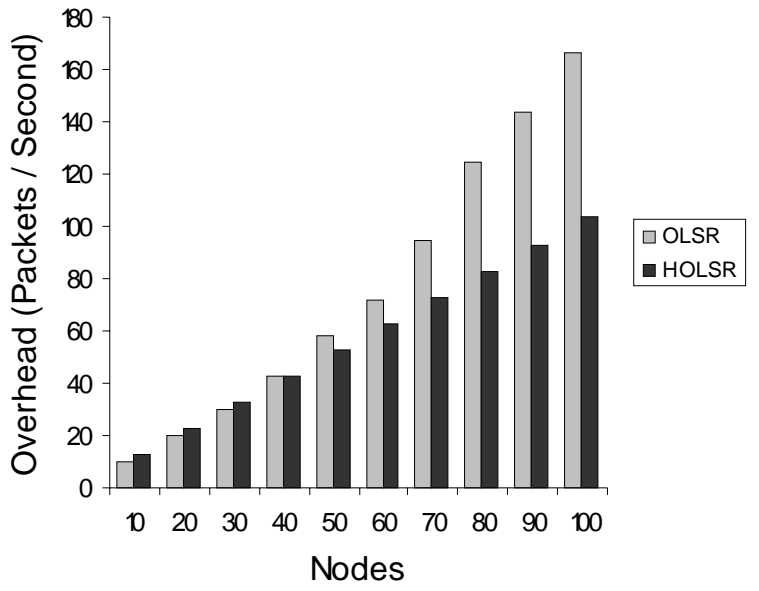

Figure 5. Overhead for OLSR and HOLSR protocols. 


\begin{tabular}{|c|c|c|c|c|c|}
\multicolumn{4}{c}{ OLSR } & \multicolumn{3}{c|}{ HOLSR } \\
\hline nodes & HELLO & TC & HELLO & TC & HNA \\
\hline $\mathbf{1 0}$ & 10 & 0 & 12,00 & 0 & 0,32 \\
\hline $\mathbf{2 0}$ & 20,04 & 0 & 22,03 & 0 & 0,35 \\
\hline $\mathbf{3 0}$ & 30,00 & 0,233 & 32,03 & 0 & 0,37 \\
\hline $\mathbf{4 0}$ & 39,57 & 2,776 & 42,00 & 0 & 0,39 \\
\hline $\mathbf{5 0}$ & 46,18 & 11,95 & 52,01 & 0 & 0,44 \\
\hline $\mathbf{6 0}$ & 54,43 & 17,63 & 61,96 & 0,05 & 0,46 \\
\hline $\mathbf{7 0}$ & 63,85 & 31,08 & 71,80 & 0,22 & 0,52 \\
\hline $\mathbf{8 0}$ & 77,06 & 47,84 & 80,38 & 1,89 & 0,64 \\
\hline $\mathbf{9 0}$ & 89,83 & 53,62 & 86,84 & 5,32 & 0,75 \\
\hline $\mathbf{1 0 0}$ & 95 & 71,69 & 93,43 & 9,24 & 0,84 \\
\hline
\end{tabular}

Table 1. Amount of routing packets for OLSR and HOLSR protocols in packets per second

Figure 5 shows that in networks with less than 40 nodes, flat routing protocols have a slightly smaller overhead of routing messages. From 40 nodes and on, the overhead in OLSR protocol grows substantially (due to TC messages generation), whereas in the hierarchical scenario, HOLSR protocol, it grows gradually. Taking into account these results, although the idea of using flat routing protocols could be considered in small networks $(<50$ nodes more or less), using hierarchical routing will produce almost the same performance. However, when the network is bigger (> 50 nodes), we find clear scalability problems in OLSR protocol, whereas in the HOLSR protocol a lineal growth is maintained in the overhead parameter. Apart from this, this routing traffic is kept within each cluster because of the use of private address allocation and aggregation. Using subnet-directedbroadcast addresses bounded by addresses with netmask, the broadcast messages will be received and correctly interpreted only by nodes within the same cluster. Due to this fact and as Table 1 shows, the amount of TC packets in medium or big scenarios is significantly smaller in HOLSR, reducing the total routing overhead.

\section{VIDEO PERFORMANCE EVALUATION}

\subsection{Simulation scenario}

The performance of the OLSR and HOLSR protocols has been evaluated with the NS-2 simulation tool with the Evalvid video evaluation tool [15] and without any mechanism of QoS. Simulation environment consists of 50 wireless nodes (moderatescale network size) forming an ad hoc network with the OLSR and an area of $1200 \times 600 \mathrm{~m}$. With the HOLSR protocol the network architecture is defined by two clusters connected by a point-to-point trunk. Radio model is the same as used in Section 3.1. Each of these clusters is defined as an area of $600 \times 600 \mathrm{~m}$. We have selected 200 seconds as simulation time.

Each node in the simulation scenario (including the source and destination) moves according to the "random waypoint" model. That is, the wireless node randomly selects a destination, moves in the direction of this location at a speed of $5 \mathrm{~m} / \mathrm{s}$, and when it arrives then pauses during the interval known as pause time. With the aim to evaluate the influence of node movement on the quality of video transmission different values are assigned in the simulations to pause time parameter: 0 s., 50 s., 100 s., 150 s. and $200 \mathrm{~s}$. A pause time of $0 \mathrm{~s}$. correspond to the worst scenario because wireless nodes are all the time moving during the simulation.

In order to evaluate the impact of the speed of the wireless nodes, we have simulated different scenarios where each node moves using random waypoint model at a $0 \mathrm{~m} / \mathrm{s}$ (all nodes are static), 5 $\mathrm{m} / \mathrm{s}, 10 \mathrm{~m} / \mathrm{s}, 15 \mathrm{~m} / \mathrm{s}$ and $20 \mathrm{~m} / \mathrm{s}$.

The video trace file used in the simulation is mobile.yuv with a size of 176x144 (QCIF) and 30 fps rate, which is available on the Internet. We have created a video repeating the same file and we have encoded the video into an MPEG-4 video trace file formed by 5098 frames, including 425 I frames, 1275 P frames and 3398 B frames. The video stream starts at 30 seconds from the beginning of the simulation.

The traffic load used consists of 20 UDP sessions with constant bit rate (CBR), established between nodes that are randomly selected. Each source sends 2 packets of 512 bytes every second (we use between medium and heavy traffic pattern) [13].

\subsection{Performance metrics}

With the aim to compare both protocols, we choose the Peak Signal to Noise Ratio (PSNR) to measure the quality of the video transmission sequence. PSNR is one of the most common objective metric used to evaluate video quality. The following equation shows the definition of the PSNR:

$$
\begin{gathered}
P S N R=10 \log _{10}\left(\frac{255^{2}}{M S E}\right) \\
M S E=\frac{1}{M N} \sum_{m=0}^{M-1} \sum_{n=0}^{N-1}\left[I_{o r g}(m, n)-I_{d e c}(m, n)\right]^{2}
\end{gathered}
$$

Where $I_{\text {org }}$ is the original image and $I_{d e c}$ is the decoded image; $\mathrm{M}, \mathrm{N}$ is the size of the image; and MSE is the Mean Square Error. The value of reference for the video trace file used is $24.37 \mathrm{~dB}$ (optimal value).

On the other hand, the performance of the video transmission using OLSR and HOLSR protocols has been evaluated in terms of packet delivery ratio. This metric is defined as the percentage of video packets successfully delivered to the destination against total packets sent.

To obtain the results, 5 simulations of each scenario have been done and average results have been presented.

\subsection{Simulation results}

In Figure 6, we present the results we have obtained with respect to the Average PSNR metric. 


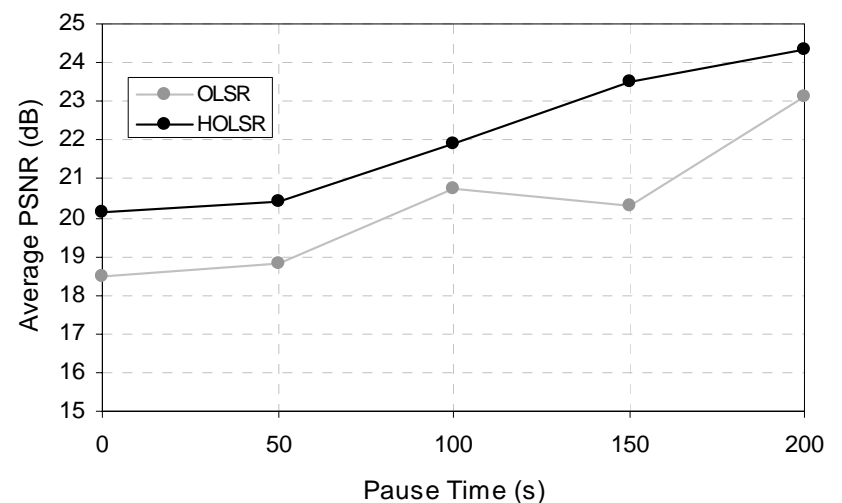

Figure 6. Average PSNR vs Pause Time.

We can observe that the Average PSNR of both protocols increased when the pause time grows. However, HOLSR improves the Average PSNR between $1 \mathrm{~dB}$ (for a pause time 200) and more than $3 \mathrm{~dB}$ (for a pause time 150, specifically, the Average PSNR is $20.32 \mathrm{~dB}$ for OLSR and 23.52 for HOLSR). Considering that we have used the same parameters when configuring scenarios for all simulations, the results obtained allow us to evaluate the improvement when the ad hoc network is configured by means of clusters and a head node is in charge of communicate the different clusters.

In Figure 7, we show the results about the ratio of delivery packets measured during the simulations. We can see that the bigger is the pause time the bigger is the percentage of frame delivered. A pause time of $0 \mathrm{~s}$. correspond to the worst scenario because wireless nodes are all the time moving during the simulation. And pause time of $200 \mathrm{~s}$. match to the best scenario when wireless nodes are all still. We can point out that as the mobility diminishes, HOLSR increases quickly the delivery ratio. For a pause time greater than 100 , the delivery ratio is up to the $80 \%$.

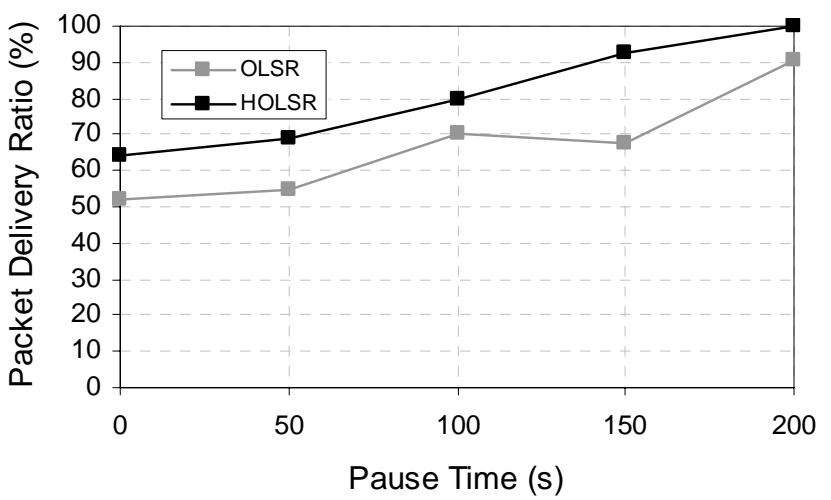

Figure 7. Packet Delivery Ratio vs Pause Time.

In Figure 8 and 9, we can see the effect of the speed of the wireless nodes.

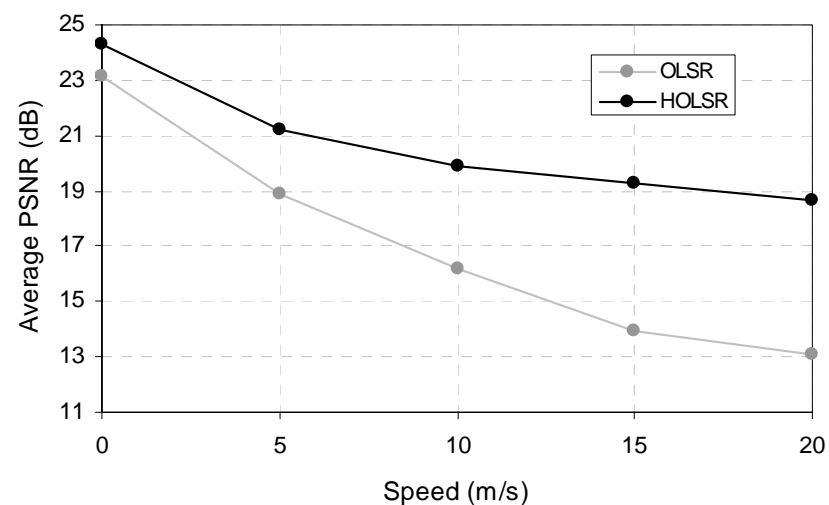

Figure 8. Average PSNR vs Speed.

As shows Figure 8, both protocols follow similar tendency to decrement the PSNR with the speed. However, OLSR seems to be very more sensible to the effects of the wireless nodes' mobility.

Figure 9 highlights the effect of the speed in the packet delivery ratio parameter. OLSR suffers a very high percentage of video packets loss. From the results it is clear that the HOLSR protocol delivers at least 10 percent more packets than the flat OLSR protocol for speed $0 \mathrm{~m} / \mathrm{s}$ and more than 40 percent more packets for $20 \mathrm{~m} / \mathrm{s}$.

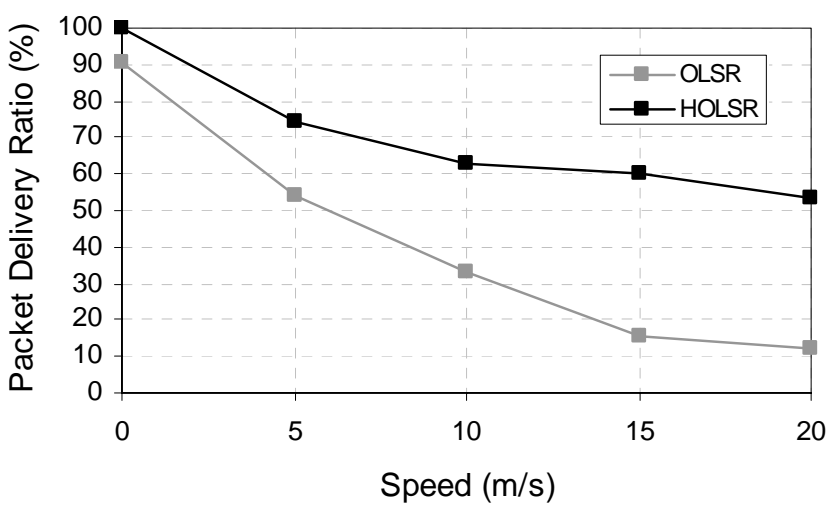

Figure 9. Packet Delivery Ratio vs Speed.

Another metric evaluated in our study is the average end-to-end delay of video packets. Figure 10 shows the performance of the routing protocols with regard to the delay parameter. This time is measured at application level, so, it includes all delays (queuing, propagation, transfer time) suffered by a packet that reaches the destination. When there is a high degree of mobility (from 0 to 100 pause time), HOLSR delivers data packets more quickly than OLSR. The main causes are the low convergence time and low overhead generated by HOLSR. Under a low degree of mobility (from 100 to 200) both protocols have similar results. Taking into account the speed of mobile nodes, simulation results (Figure 11) show that HOLSR has always better performance than OLSR. HOLSR has the shortest delay (no more than $50 \mathrm{~ms}$ ). 


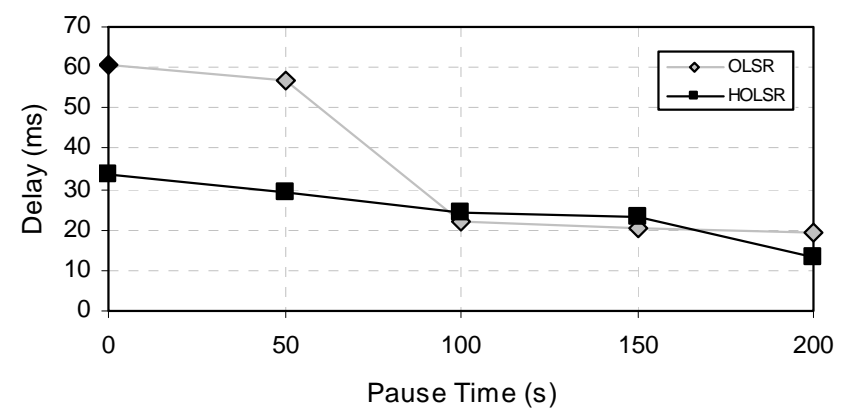

Figure 10. Delay vs Pause Time.

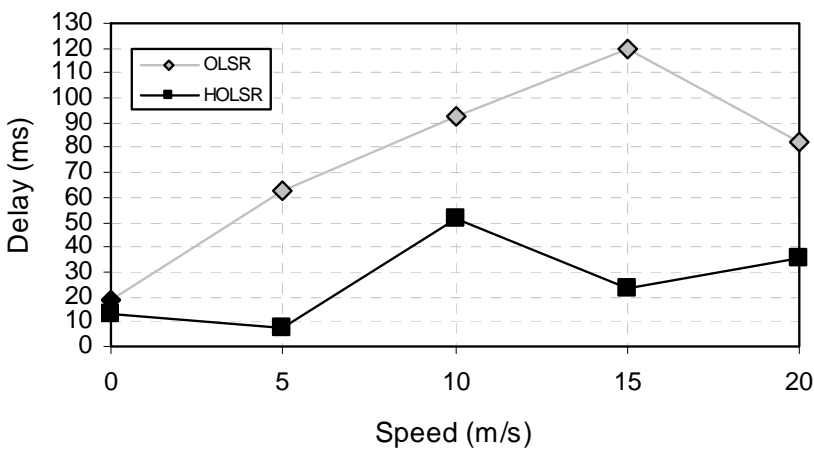

Figure 11. Delay vs Speed.

\section{CONCLUSIONS AND FUTURE WORK}

A lot of works has been done in ad hoc networks, taking into account different scenarios and data traffic conditions with the aim to evaluate different routing protocols. But, there is not many works about hierarchical protocols and specifically to evaluate video traffic quality. In this paper, we have done a study considering a well known flat protocol (OLSR) and a new algorithm based on the hierarchical protocol (HOLSR). The main conclusion is that HOLSR is a good candidate for video transmission over ad hoc networks. This protocol has good PSNR and frame loss performance (taking into account that we are not using any QoS mechanism).

As future work, we plan to study and evaluate how to reduce further the overhead using several levels in the hierarchical network; how to select the best head node; and how to include QoS mechanism in hierarchical networks to guarantee the performances of the real time communications. Finally, we plan to evaluate subjective quality of received videos making tests for this purpose.

\section{ACKNOWLEDGMENTS}

We would like to acknowledge the European Commission for their support. Our acknowledgement goes also to all ADHOCSYS partners for their co-operation and contribution in this project.

\section{REFERENCES}

[1] Basagni, S., Conri, M., Giordano, S., and Stojmenovic, I. Mobile ad hoc networking. John Wiley \& Sons (IEEE Press), 2004.
[2] Ramanathan, S., and. Steenstrup, M.E. A survey of routing techniques for mobile communications networks, Mobile Networks and Applications (ACM/Baltzer), Volume 1, (1996), 89-104.

[3] Boukerche, A. Performance evaluation of routing protocols for ad hoc wireless networks. Mobile Networks and Applications (Kluwer Academic), Volume 9, Issue 4 (August 2004), 333-342.

[4] Haerri, J., Filali, F., and Bonnet, C. Performance comparison of AODV and OLSR in VANETs urban environments under realistic mobility patterns. In Proceedings 5th IFIP Mediterranean Ad-Hoc Networking Workshop, June 14-17, 2006, Lipari, Italy.

[5] Pore Ghee Lye Mceachen, J.C., A Comparison of Optimized Link State Routing with traditional routing protocols in Marine Wireless Ad-hoc and Sensor Networks. In Proceedings of International Conference on $40^{\text {th }}$ Annual Hawaii, Jan. 2007

[6] Clausen, T., and Jacquet, P. Optimized Link State Routing Protocol (OLSR), RFC 3626, October 2003.

[7] Murthy, S. and Garcia-Luna-Aceves, J.J. An efficient routing protocol for wireless networks. Mobile Networks and Applications, 1 (1996), 183-197.

[8] Park, V.D. and Corson, M.S. A highly adaptive distributed routing algorithm for mobile wireless networks. In Proceedings of IEEE INFOCOM'97, Kobe, Japan (April 1997) pp. 1405-1413.

[9] Perkins, C.E. and Bhagwat, P. Highly dynamic destination sequenced distance vector routing (DSDV) for mobile computers. In Proceedings of ACM SIGCOMM'94, London, UK (August-September 1994), 234- 244.

[10] Perkins, C.E., and Royer, E.M. Ad hoc on-demand distance vector (AODV) routing. RFC 3561, July 2003.

[11] Zhao, S. et al. Routing protocols for self-organizing Hierarchical Ad Hoc Wireless Networks. IEEE Sarnoff Symp., Trenton, NJ, Mar. 2003.

[12] Villasenor-Gonzalez, L., Ge, Y., and Lamont, L., HOLSR: A Hierarchical Proactive Routing Mechanism for Mobile Ad Hoc Networks, IEEE Communications Magazine (Vol 43, No. 7, July 2005), 118-125.

[13] Ge, Y., Lamont, L., and Villasenor, L. Hierarchical OLSR -A Scalable Proactive Routing Protocol for Heterogeneous Ad Hoc Networks. In Proceedings of the Wireless and Mobile Computing, Montreal (WiMob 2005), (Canada, August 22-24, 2005), 17-23.

[14] Kao, K. L., Ke C. H., and Shieh C. K. Video Transmission Performance Evaluation of Ad Hoc Routing Protocols. In Proc. Intern. Conf. on Intelligent Information Hiding and Multimedia Signal Processing (IIH-MSP'06), (Pasadena, California, USA, Dec. 18-20, 2006. IEEE Computer Society, 181-184.

[15] Ke, C.H., Lin, C. H., Shieh, C. K. and Hwang, W.S. A novel realistic simulation tool for video transmission over wireless network. In Proceedings of the IEEE International Conference on Sensor Networks, Ubiquitous, and Trustworthy Computing (SUTC2006), June 5-7, 2006. 